NSF-ITP-93-154

Dec. 1993

\title{
PROPAGATION OF ELECTROMAGNETIC WAVES IN THE CORE OF A STRING DEFECT IN LIQUID CRYSTALS
}

\author{
Ajit M. Srivastava and Michael Stone® \\ Institute for Theoretical Physics, University of California \\ Santa Barbara, California 93106, USA
}

\begin{abstract}
We investigate the propagation of electromagnetic waves in the core of a winding number one string defect with isotropic core in nematic liquid crystals. We numerically solve wave equations for the TE mode and show the existence of guided modes arising due to the variation of the refractive index as a function of the scalar order parameter.
\end{abstract}

\footnotetext{
${ }^{1}$ Physics Department, University of Illinois at Urbana Champaign, 1110 W. Green St. Urbana, Illinois 61801, USA
} 


\section{INTRODUCTION}

Optical fiber waveguides have been a subject of intense theoretical and experimental investigations due to their technological importance. Propagation of electromagnetic waves in such waveguides has been analysed in detail for different waveguide geometries as well as for different optical properties of the materials used. The simplest optical fiber essentially consists of an inner cylindrical core of a transparent medium with refractive index $n_{1}$ surrounded by an outer cladding of another transparent material with refractive index $n_{2}$ such that $n_{1}>n_{2}$ [洒]. There are also graded index fibers where the refractive index decreases continuously in radial direction [2]. Recently it has been suggested that the propagation of electromagnetic waves in the cores of string defects in certain transparent condensed matter systems may show interesting behavior and that these strings may behave as graded optical fiber waveguides [3]. However, the considerations in [3] were based on geometrical optics notions which are not very appropriate for the realistic cases when the thickness of the string defects is of the order of the wavelength of light used.

In this paper we analyze in detail the propagation of electromagnetic waves in the core of a winding number one string defects with isotropic cores in nematic liquid crystals. Optical fibers with liquid crystalline cores have been discussed in the literature by Lin et al. [4, 5] and we follow the approach used in [4] in our investigation. Our work differs from that in [4] in two important aspects. The propagation of light was considered in [4] for the case when the liquid crystalline core was entirely in the nematic phase (apart from the consideration of the effects of the propogating fields on the order parameter as discussed in (5]), even though the configuration of the director field considered in [4] corresponded to strength one line defect. The reason being that for physically interesting wavelengths, the cylindrical cavity had to be large making it energetically unfavorable for the strength one defect to develop an isotropic core. We, however, consider the case when the core of the string is in the isotropic phase. This leads to radially decreasing value of

the refractive index for the TE mode (as we will see later) resulting in the existence of guided modes even without a transparent cladding. This is the second difference between our work and that in [4] where guided modes were due to the lower value of the refractive index in the cladding material. Even though our consideration of the isotropic core for the string restricts us to a wavelength regime which is probably not much of practical use (in the sense that absorption of light may be strong for such short wavelengths), it illustrates a novel possibility where the guided modes arise purely due to the variation 
of the order parameter field. As the order parameter field varies continuously, we expect the refractive index to do the same (as we will explain below) making these strings into graded optical fibers.

\section{WAVE PROPAGATION}

We consider a cylindrical waveguide consisting of nematic liquid crystalline core at a temperature below the nematic-isotropic transition temperature. The walls of the cylinder (which need not be transparent) are assumed to be coated with appropriate material so that the director is anchored normal to the wall leading to a strength one string configuration [6]. We further assume that the radius of the cylinder is small enough so that at the center of the cylinder the order parameter vanishes leading to an isotropic core for the string. The conditions under which this is energetically favorable have been analysed in [4] and we refer the reader to [4] for further details. The director then lies in the plane perpendicular to the axis of the cylinder and points radially from the center. We will consider the wave propagation only near the core of the string and hence neglect any possible surface effects.

The radial variation of the order parameter $S$ for a strength one string defect (in the single constant approximation) is governed by the Landau-de Gennes free energy density

$$
F=\frac{A}{2} S^{2}-\frac{B}{3} S^{3}+\frac{C}{4} S^{4}+\frac{L S^{2}}{2 r^{2}}+\frac{L}{2}\left(\frac{\partial S}{\partial r}\right)^{2}
$$

Here we have omitted the surface contribution as we are only interested in the idealized case of the wave propagation near the core of the string. Here $A=a\left(T-T_{0}\right), T$ is the temperature and $a, T_{0}, B, C$ and $L$ are parameters of the material. We take the values of these parameters [4, 7] as, $a=0.1319 \times 10^{5} \mathrm{~J} / \mathrm{m}^{3} \mathrm{~K}, B=1.836 \times 10^{5} \mathrm{~J} / \mathrm{m}^{3}, C=$ $4.050 \times 10^{5} \mathrm{~J} / \mathrm{m}^{3}, L=2.7 \times 10^{-11} \mathrm{~J} / \mathrm{m}$ and $T_{0}=307 \mathrm{~K}$. With these parameters, the string profile can be obtained from the following equation corresponding to the above free energy

$$
\frac{d^{2} S}{d r^{2}}+\frac{1}{r} \frac{d S}{d r}-\frac{S}{r^{2}}-\frac{1}{L}\left(A S-B S^{2}+C S^{3}\right)=0
$$

We have solved the above equation numerically using a Runge-Kutta algorithm of fourth order accuracy. The boundary conditions for the order parameter are that $S(r)$ vanishes at $r=0$ (corresponding to the isotropic phase) and at large $r, S(r)$ approaches its asymptotic value $\eta$ corresponding to the nematic phase. The profiles of $S(r)$ we obtain 
for various temperatures are in agreement with those in [四. We now try to determine the spatial variation of the refractive index for this configuration and whether it can lead to the existence of guided modes. At the center of the string the refractive index should have the value $n_{i}$ appropriate for the isotropic phase. At large $r$, in the nematic phase, there are two indices of refraction; $n_{\|}$for the extra-ordinary ray and $n_{\perp}$ for the ordinary ray. We will consider the propagation of TE mode for which the $n_{\perp}$ is the appropriate refractive index for the nematic phase. As we have mentioned earlier, guided TE modes arise here due to the fact that the refractive index decreases from the value $n_{i}$ at the center of the string to a smaller value $n_{\perp}$ away from the center.

We now assume that the dielectric permittivity $\epsilon_{\perp}$ for the ordinary ray is proportional to the order parameter $S$ [5]. With the boundary conditions that $\epsilon_{\perp}(S=0)=n_{i}^{2} \epsilon_{0}$ and $\epsilon_{\perp}(S=\eta)=n_{\perp}^{2} \epsilon_{0}$, we can derive the spatial dependence of $\epsilon_{\perp}(r)$ resulting from the spatial dependence of $S(r)$,

$$
\epsilon_{\perp}(r)=\epsilon_{0}\left[n_{i}^{2}-\frac{\left(n_{i}^{2}-n_{\perp}^{2}\right)}{\eta} S(r)\right]
$$

where $\epsilon_{0}$ is the permittivity of the vacuum.

To study the propagation of electromagnetic waves in the waveguide we consider the Maxwell's equations

$$
\begin{aligned}
\nabla \times \mathbf{E} & =-\frac{\partial \mathbf{B}}{\partial t} \\
\nabla \times \mathbf{H} & =\frac{\partial \mathbf{D}}{\partial t} \\
\nabla \cdot \mathbf{D} & =0 \\
\nabla \cdot \mathbf{H} & =0
\end{aligned}
$$

Where,

$$
\begin{aligned}
& \mathbf{D}=\epsilon \mathbf{E} \\
& \mathbf{B}=\mu_{0} \mathbf{H}
\end{aligned}
$$

here $\mu_{0}$ is the permeability of the vacuum (we will assume this to be the same for the medium as well) and $\epsilon$ is the permittivity of the medium.

We consider the propagating modes in the following form

$$
\mathbf{E}(r, z, \phi)=\mathbf{E}(r) e^{i(\omega t-\beta z)}
$$


with similar $(z, t)$ dependence for $\mathbf{H}$ as well. $\omega$ and $\beta$ are the optical frequency and propagation constants respectively. Uncoupled equations for field components can be obtained From Eqns.(4)-(9) for the TE mode by taking $\mathbf{E}=\left(0, E_{\phi}, 0\right)$ and $\mathbf{H}=\left(H_{r}, 0, H_{z}\right)$. We assume that the director varies uniformly with the azimuthal angle. Since $E_{\phi}$ is always normal to the director, the permittivity $\epsilon_{\perp}$ only depends on $r$ and is given by Eqn.(3). We get,

$$
\begin{gathered}
\frac{\partial^{2} E_{\phi}}{\partial r^{2}}+\frac{1}{r} \frac{\partial E_{\phi}}{\partial r}+\left[\omega^{2} \mu_{0} \epsilon_{0}\left\{n_{i}^{2}-\frac{\left(n_{i}^{2}-n_{\perp}^{2}\right)}{\eta} S(r)\right\}-\beta^{2}-\frac{1}{r^{2}}\right] E_{\phi}=0 \\
H_{r}=-\frac{\beta}{\omega \mu_{0}} E_{\phi} \\
H_{z}=\frac{i}{\omega \mu_{0}}\left(\frac{\partial E_{\phi}}{\partial r}+\frac{E_{\phi}}{r}\right)
\end{gathered}
$$

Here we have replaced $\epsilon$ by $\epsilon_{\perp}$ from Eqn.(3) as appropriate for the TE mode. Using $\epsilon_{0} \mu_{0} \omega^{2}=(2 \pi / \lambda)^{2}$ and writing $\beta$ as $2 \pi / \lambda_{z}$, we can rewrite the above equation as

$$
\begin{aligned}
\frac{\partial^{2} E_{\phi}}{\partial r^{2}}+\frac{1}{r} \frac{\partial E_{\phi}}{\partial r} & +\left[A-B S(r)-\frac{1}{r^{2}}\right] E_{\phi}=0 \\
A & =4 \pi^{2}\left[\frac{n_{i}^{2}}{\lambda^{2}}-\frac{1}{\lambda_{z}^{2}}\right] \\
B & =\frac{4 \pi^{2}}{\eta \lambda^{2}}\left[n_{i}^{2}-n_{\perp}^{2}\right]
\end{aligned}
$$

We may mention here that guided TM modes will not be expected to arise for the case under consideration. This is because for such modes $E_{r}$ does not vanish and for $E_{r}$ the corresponding refractive index $n_{\|}$in the nematic phase, away from the string center, is larger than the value $n_{i}$ at the center.

\section{NUMERICAL SOLUTIONS}

We solve Eqn.(2) for various values of temperatures and use the resulting profile of $S(r)$ in the expression of $\epsilon_{\perp}(r)$ to solve for $E_{\phi}$ in Eqn.(14). Numerical solutions are again obtained by using a Runge-Kutta algorithm of fourth order accuracy. Boundary conditions for $E_{\phi}$ are that $E_{\phi}=0$ at $r=0$ and at $r \rightarrow \infty$ (for guided mode). We choose a fixed value of $B$ and vary $A$ to find the solution of Eqn. (14) which is well behaved at large $r$. We then decrease the value of $B$ to search for the largest value of the wavelength $\lambda$ for which a guided mode can be found. Values of $\lambda$ and $\lambda_{z}$ can be obtained from the values of $A$ and $B$ from Eqns. (15)-(16). Profiles of $H_{r}$ and $H_{z}$ are obtained from Eqns(12)-(13). 
We take the values of the indices of refraction as, $n_{i}=1.6$ and $n_{\perp}=1.5$ [4]. It is important to mention here that these values of refractive indices are typical at optical wavelengths while the wavelengths relevent for our case come out to be much shorter. Though, we note that numerically we obtain the value of $A$ and $B$ and the refractive indices are used only to determine the corresponding values of $\lambda$ and $\lambda_{z}$ from Eqns.(15)(16). We take the above values of refractive indices as an example. Thus, if the value of $n_{i}^{2}-n_{\perp}^{2}$ is larger for shorter wavelengths then the corresponding wavelength will also be larger. [As we mentioned earlier, for short wavelengths the absorption of light may also be strong. We will, however, not worry about this. Our main aim here is to illustrate the qualitative feature of the existence of guided modes purely due to the spatial variation of the order parameter.]

Fig. (1) shows the plots of $E_{\phi}$ and $H_{z}$ for different temperatures and for different values of $\lambda . H_{r}$ can be simply obtained from Eqn.(13). Our choice of temperatures was guided by the consideration of finding largest values of the core radius of the string which would lead to large, possibly practically more interesting, values for the wavelength $\lambda$. The most favorable value of $\lambda$ we could find for our set of parameters is about $2670 \AA$ corresponding to the plot shown in Fig. 1a.

\section{CONCLUSIONS}

We have shown the existence of guided modes for a strength one string configuration with isotropic core due to the variation of the refractive index as a function of the order parameter. Even though the consideration of isotropic core has restricted as to the consideration of wavelengths of the order of $2600 \AA$, we believe that our results point out an interesting possibility that for suitable liquid crystal samples for which the string size can be made larger, guided modes can be found purely within the liquid crystalline core as compared to the cases where the lower refractive index of the cladding is responsible for guided modes. Further, due to continuous dependence of the refractive index on the order parameter, the resulting waveguide is similar to a graded index fiber.

\section{ACKNOWLEDGEMENTS}

We would like to thank M. Lee and P. Palffy-Muhoray for providing ref. [7]. This work was supported by the National Science Foundation under grant numbers PHY89-04035 and DMR91-22385. 


\section{References}

[1] L.B. Jeunhomme, "Single-mode fiber optics", (Marcel Dekker, Inc., New York, 1990).

[2] G.H. Owyang, "Foundation of optical waveguides", (Elsevier North Holland, Inc., 1981); W.A. Gambling and H. Matsumura, Opt. Quant. Elect. 10, 31 (1978); D. Marcuse, J Opt. Soc. Am. 68, 103 (1978).

[3] A.M. Srivastava, ITP, University of California preprint, TPI-MINN-92/53-T, NSFITP-92-145.

[4] H. Lin, P. Palffy-Muhoray and M. A. Lee, Mol. Cryst. Liq. Cryst. 204, 189 (1991); H. Lin and P. Palffy-Muhoray, Opt. Lett. 17, 722 (1992).

[5] H. Lin and P. Palffy-Muhoray, Liq. Cryst. 14, 1977 (1993).

[6] P.E. Cladis, Philos. Mag. 29, 641 (1974).

[7] D.W. Allender and S. Zumer, SPIE Proceesings, Vol. 1080, 18 (1989). 


\section{FIGURE CAPTIONS}

(1) Profile of the order parameter $S(r)$ is shown by the solid curve while $E_{\phi}$ and $\frac{1}{2} H_{z}$ are shown by the dashed and the dotted curves respectively. (a) Plots for $\triangle T=1.44$

and $\lambda=2677 \AA$. $\lambda_{z}$ is found to be $=1790 \stackrel{\circ}{A}$. (b) Plots for $\triangle T=1.35, \lambda=1420 \AA$ and $\lambda_{z}=945 \stackrel{\circ}{\text {. }}$ 